\title{
Less is More: Classifying Mobile Interactions to Support Context Sensing in Journeys
}

\author{
Claudia Krehl \\ The University of Nottingham \\ Horizon Doctoral Training Centre \\ psxck@nottingham.ac.uk
}

\author{
Sarah Sharples \\ The University of Nottingham \\ Human Factors Research Group \\ sarah.sharples@nottingham.ac.uk
}

\author{
Martin Flintham \\ The University of Nottingham \\ Horizon \\ martin.flintham@nottingham.ac.uk
}

\begin{abstract}
This paper develops a classification of mobile interactions based on contextual information relevant to the mobile device user in journeys. Context-aware systems can be used to reduce the stress involved, support users in their activities and increase utility of travel time. But context is often portrayed as real, stable and structured, which can limit the value of applications as they lack dynamics and relevancy. This paper aims to classify mobile interactions in journeys by adopting an alternative view of context. It is argued that sensing less contextual information can be more valuable providing the most relevant information to the user can be identified. Context is explored using qualitative approaches that investigate user interactions during end-to-end journeys. The resulting classification serves as a basis for understanding mobile interactions and it assists designers and $\mathrm{HCl}$ practitioners to develop improved context-aware application.
\end{abstract}

Mobile Interactions. Context. Task. Social Setting. Journeys.

\section{INTRODUCTION}

Human-computer interactions in mobile settings have an integral role in our personal and professional life. Mobile devices provide users with ubiquitous information access, entertainment and they help users to stay connected to work, friends and family [9]. Lately, mobile devices interactions have changed significantly. Users carry out a wider range of tasks, and the nature of tasks has changed due to increased functionality. Users are also faced with a number of challenges when interacting in mobile environments such as the need or desire for multitasking, management of interruptions or unexpected events, and additional distractions or the fluctuation of settings [1].

Studying interactions in journeys offers researchers with opportunities to understand these concepts. When travelling people often multitask and carry out a variety of tasks including communications, entertainment or recreation, household or personal activities while coping with the challenges mobility poses [12]. Above all researchers can investigate the strategies and behaviour people adopt in irregular journeys. Users face more decisions and uncertainty due to the lack of a routine or habit [13]. As an ever increasing amount of time is spent travelling it is key to support this time effectively to increase positive travel time utility. Kenyon \& Lyons [12] also suggest that the ability to multitask can influence the attractiveness of travel and as an enabler for the secondary task. To achieve this via context-aware systems we need to understand how the context of a journey shapes the behaviour users adopt when interacting with devices.

Two key observations can be made about the role of context for mobile interfaces. Firstly, mobile interaction design cannot be separated from context, as it influences actions and how people interpret and understand these [6]. But the current approach of viewing context as real, structured and stable is impractical [15]. Instead it could be viewed as constantly changing in terms of the information that is relevant to individuals and the contextual information itself. Thus, there is a need for context to be examined using an approach closer to social science, where context is treated dynamically within a frame of reference and most importantly as actively created and managed by users [7].

Finally, context-aware systems that use "context to provide relevant information and/or services to the user, where relevancy depends on the user's task" $[4$, p.5] also create opportunities to improve the user experience during mobile interactions. Context-aware applications have been developed in many areas such as reminder services or information provision [e.g. 5; 19]. But most of these rely on information that relates to the approach that treats contexts as real, structured and stable, so it may or may not be relevant to the user. Hence, the value and impact these services have on the user's behaviour for instance when travelling are limited. There is a need to show whether dynamic elements 
of context have a larger effect on user behaviour. If so, this type of contextual information could be used to increase the positive utility while travelling.

These observations point to the need for a classification of context that addresses mobile interactions in journeys. Therefore this paper aims to develop a classification of mobile interactions in journeys by critically analysing the value of all components of context instead of focusing on static elements of context only. The findings can help designers, $\mathrm{HCl}$ practitioners and researchers to create context-aware services of higher impact and this higher value to the user experience.

\section{BACKGROUND}

\subsection{Using Contextual Information}

The notion of context has been widely discussed in the literature. Generally speaking context involves circumstances, backgrounds and settings [2]. However, such a definition is very broad. Dey [4, p. 5] provides a more compelling definition describing context as "any information that can be used to characterise the situation of an entity. An entity is a person, place, or object that is considered relevant to the interaction between a user and an application including the user and applications themselves." Five distinct elements of contexts, namely physical, temporal, social, technical, and task contexts, and their features were identified in a comprehensive review [11] as shown in Table 1.

Considering all aspects of context, it becomes clear that context can be defined by a large number of factors in a myriad of detail. However, simply trying to gather as much contextual information as possible can be problematic. First, the information that can potentially be collected about a user's context could be far too granular as the knowledge of what is actually important to the user in that situation can be lost. This reduces the impact on interactions. Bradley \& Dunlop [2] also argue that the notion of relevancy is vital when endeavouring to comprehend human behaviour. For instance, sensors can detect weather, acceleration, position, networks, calendar entries, contacts, other devices, and so on, but whether this information is relevant to the user at that point is not clear. As researchers

Table 1: Elements of Context

\begin{tabular}{|l|l|}
\hline \multicolumn{1}{|c|}{ Element } & \multicolumn{1}{c|}{ Description } \\
\hline Physical & Location, Altitude, Physical Objects \\
\hline Social & $\begin{array}{l}\text { Presence of others, Roles \& } \\
\text { Responsibilities, Interactions }\end{array}$ \\
\hline Task & User task, Multitasking, Interruptions \\
\hline Technical & $\begin{array}{l}\text { Availability of Infrastructure, Capability } \\
\text { of Soft- \& Hardware, Sensors }\end{array}$ \\
\hline Temporal & $\begin{array}{l}\text { Time, Past \& future events, hurrying or } \\
\text { waiting }\end{array}$ \\
\hline
\end{tabular}

and $\mathrm{HCl}$ practitioners we should not be asking "What can we sense?" but "What should we sense?" to collect information that is meaningful to the user. Thus some contextual information should be regarded as background and some as being of selective interest, which can enrich user knowledge or can be used as a resource to carry out tasks.

This is further supported by Dourish [7] who argues that the notion of relevancy is dynamic. Users determine ad hoc and in situ what information is significant. So relevancy is an emergent attribute of an interaction and it is thus vital to investigate how context actively shapes relevancy. The most influential study carried out in this area was conducted by Tamminen et al. [19] who identified five characteristics of mobile contexts that shape user interactions, which are essentially related to task, social and temporal context. First, users pursue a goal but sidestepping can take place to fulfil situational acts due to unplanned context changes. Secondly, multitasking is viewed as a challenging undertaking, especially when navigating or monitoring the completion of sub goals. In terms of the social context, users tend to claim personal or group spaces that regulate the users' social interactions. Finally, temporal tensions were identified, which are shaped by fluctuations in the importance of time and space.

The second problem with gathering large quantities of contextual information is linked to the different philosophical approaches that relate to definitions of the notion of context. Traditionally it is shaped around Realism, which believes that there are properties, so called universals, which can be applied to many things [15]. Here, context exists, and therefore computers can recognize contextual information and adapt to it. Thus context is viewed as a representational problem [7]. But social scientists view context in light of Constructivism. Context does not reflect absolute truths, but it is subject to convention, human perception, and social experience [15]. Hence, contexts are actively created and technology should provide support for managing them. So context becomes an interactional problem due to its' relational property that holds between subjects and activities, so it is not simply a question of existence, but one of relevance to a particular activity because it is negotiated and contested and it is contingent on interpretation and reinterpretation [7].

The literature reveals a focus on physical, temporal and technical aspects, all of which are fairly static descriptors of context [11]. However, the alternative view suggests that the dynamic aspects, such as the task and social elements, are more relevant for interactions as they shape the user context. Or as Dourish argues, the "importance of context is not what it is but what it does in interaction - the role that it plays and the ways in which it is sustained 
and managed" [7, p. 28]. This view is evidently more suitable when dealing with mobility due to the ever changing nature of context and its' relevancy to the user during the time of interaction. A study on mobile information needs support this by revealing that context prompted interactions $72 \%$ of the time and activity, conversations, location and time were relevant categories of context [17].

This paper adopts the alternative philosophy which focuses on identifying elements of the environment relevant to interactions in mobile settings and on how context is actively created by users. Contextaware systems can then harness the most relevant information to effectively influence interactions.

\subsection{Building Context-Aware Systems}

Context-aware systems aim to create new forms of interactions by enhancing physical environments with technology that can respond to the needs and activities of the people within them [6]. Various context-aware applications have been developed, but opportunities to increase their value remain. Some use location information for a reminder service [5] or to displays pictures to users at opportune moments [20]. While location is a particularly popular method of sensing, other physical elements are also used. For instance, audio cues can be sensed to infer location [18]. Effects of photos taken by context camera are affected by sound and movement [10].

Yet it is much harder to find applications that infer social or task context. Shin et al. [16] developed a dynamic home screen that only displayed the applications the user is most likely to use given a context. And Esbjörnsson et al. [8] describe a system that automatically shares HTML documents with peers when passing each other.

Applications are generally tailored for specific cases, rather than tackling a set of interactions. But context-aware systems that manage a number of activities like calling, messaging, emails, searches, etc. to improve the overall user experience are valuable too. When travelling this could reduce stress and increase travel time utility. The use of social and task elements as a basis for contextaware systems are overlooked frequently in contrast to other elements. These flaws can be addressed by developing a classification of mobile interactions which shows the relevance of particular elements of context to mobile interactions.

\subsection{End-to-End Journeys}

End-to-end journeys are an ideal setting for understanding the behaviour of users when engaging with their mobile devices. A variety of secondary tasks are combined with travelling tasks. These include communications, entertainment or recreation, household or personal activities [12].
Thus, journeys allow researchers to gain an overview of behaviour in multitasking situations: are activities dependent on certain settings, and is behaviour influenced when these change?

Travel time is mostly regarded as a waste of time, but studies show that travelling can have positive uses. Three elements can cause positive utility in travel [14]. Activities conducted at the destination can lead to a halo effect. Utility can also be gained via the activities during travel such as anti-activities like getting quiet time and people gazing or engaging in activities like reading or working. The activity of travel itself can also be positive. In the commute positive utility is generally easier to achieve as established habits and routines allow the individual to focus on other activities. This is less so for infrequent journeys as uncertainty prevails [13]. Thus, this paper focuses on unfamiliar end-to-end journeys where the travel activities are more pertinent and supporting them via contextaware applications could reduce stress associated with these and increase the positive utility gained from using travel time otherwise.

This paper therefore builds a classification of mobile interactions by adopting a dynamic view of context in end-to-end journeys. The classification helps create context-aware interfaces to improve user experiences. A number of assumptions are made that guide the observation procedure. Firstly, multitasking is believed to be prominent in mobile environments encountered while travelling, leading users to develop priorities to manage tasks and interactions according. Secondly, the task and social elements of context play a central role, whereas technical, physical and temporal elements are likely background information.

\section{DEVELOPING A CLASSIFICATION OF MOBILE INTERACTIONS}

Direct and directed observation was chosen to classify mobile interactions in end-to-end journeys for two reasons. It enables the capture of rich, reallife data about contexts in which users were engaging with their personal mobile devices [9]. As this research is interested in contextual and behavioural aspects, observation data can provide valuable insights while ecological validity is maintained. Observations also have an advantage over interviews and diary studies as they do not rely on self-report. However, the observer can only see what is happening but not why. So, interviews were performed afterwards to appreciate some of the behaviour displayed and decisions made.

\subsection{Participants}

The observation was carried out with 20 participants, 9 male and 11 female participants, 
with an average age of 28.3. Participants were recruited via email invitations and face-to-face encounters. All participants were observed on their journeys to and from an event of their choice.

\subsection{Observation Framework}

The observation scheme covers three aspects to understand mobile interactions in journeys. To provide an overview of mobile interactions, basic interaction data including information on journey stages, task occurrence, time, length, sequences, performance, and mobile devices used is collected. Secondly, five elements of context, i.e. the social, temporal, physical, technical, and task context were recorded to determine the extent by which these factors influence interactions. Finally, actions individuals carried out were covered in detail to help uncover how context influences interactions by gaining insights on the process of managing and completing current tasks within that setting. Tasks were given special attention as they represent the dynamic part of context for mobile interactions.

\subsection{Procedure}

Observations were carried out during journeys to and from events chosen independently by each participant. For the purpose of this research, an event is defined as a non-frequent journey for personal or professional reasons, which is not carried out on a daily basis. The participants were met at their start location before the journey, where they gave consent and filled in a questionnaire. During the journey, any interactions between the participant and his/her personal mobile device(s) were observed using the pre-defined observation scheme to guide data collection. Photos were taken to capture further details of the interaction. A few days after the observation, a short follow-up interview was carried out, covering general usage patterns of mobile devices and specific points of interest that arose during the observation period.

\subsection{Analysis}

Content analysis was performed on the observation data for each interaction using open coding and a feedback loop [3]. The analysis focussed on tasks and social contextual information and how these change over time. The literature showed gaps in this branch of research as these contextual factors which represent the more dynamic aspects of context are underrepresented in context-aware applications and studies of mobile interactions. Tamminen et al. [19] also showed the importance of these factors. Thus, temporal, physical, and technical elements were given less attention.

After establishing what categories tasks and social situations fall into, a spread sheet was used to investigate how active behaviour was influenced by
Table 2: Context Classifications

\begin{tabular}{|c|l|}
\hline $\begin{array}{c}\text { Task Context } \\
\text { Classification }\end{array}$ & $\begin{array}{l}\text { Mobility Essential, Mobility } \\
\text { Desired, Goal Essential, Goal } \\
\text { Desired, Entertainment }\end{array}$ \\
\hline $\begin{array}{c}\text { Social Context } \\
\text { Classification }\end{array}$ & $\begin{array}{l}\text { Alone, With friends \& family, } \\
\text { With colleagues }\end{array}$ \\
\hline
\end{tabular}

these elements of context by exploring the relations between task and social context and the activities of participants during event end-to-end journeys. Five task categories and three social categories were identified, as shown in Table 2.

\subsection{Results}

In total 172 mobile interactions were recorded and individual journey times ranged from 9 minutes to 5 hours and 15 minutes. A series of mobile devices were used such as SatNavs, mobile phones, smart phones, MP3 players, laptops, etc. Interactions also occurred on a number of different modes of transport including walking, travelling on a bus, train or car, and driving. Participants travelled to a variety of events for both personal and professional reasons. The observations showed that three categories of context influenced the activities and behaviour of users in mobile device interactions task, social and location aspects of context. But the participants easily adjusted to changes in physical, temporal and technical aspects of context. In terms of task context, five task types were identified. Firstly, mobility essential tasks incorporate activities whose goals are related to the journey and they are related to the mobile setting. The completion of the journey depends on their execution. Activities that are linked to the journey but are not required for its completion are called mobility desirable tasks. During goal essential tasks an individual seeks to complete a goal, unrelated to the journey, driven by an inner motivation of that individual. Similarly, goal desired tasks pursue a goal but there is no time pressure to complete the task. Finally, tasks that pursue no goal per se, apart from relaxation or passing time, are classed as entertainment tasks. Any one task, particularly cognitive activities such as talking, could fall into a variety of categories depending on the goal. Example activities and occurrence rates are shown in Table 3.

Moreover, multitasking occurred in 140 (81\%) of the observed interactions. Some participants employed subliminal or deliberate coping strategies when multitasking such as participants slowing down their walking pace in 21 observations and 16 further observations also showed people reducing their workload by using their devices briefly when they had a quiet moment. But on some occasions participants failed to multitask. For instance, on 31 occasions participants stopped their conversations in favour for interaction tasks. On 8 occasions (5\%) participants even failed to complete the activity. 
Table 3. Task Examples and Occurrence Rate

\begin{tabular}{|l|l|l|c|}
\hline \multicolumn{1}{|c|}{$\begin{array}{c}\text { Task } \\
\text { Name }\end{array}$} & \multicolumn{1}{|c|}{ Definition } & \multicolumn{1}{|c|}{ Example Activities } & $\begin{array}{l}\text { Rate } \\
(\%)\end{array}$ \\
\hline $\begin{array}{l}\text { Mobility } \\
\text { Essential }\end{array}$ & $\begin{array}{l}\text { Goal is related to journey. Completion is } \\
\text { essential for journey completion. }\end{array}$ & $\begin{array}{l}\text { Walk, Drive, Talk, Navigation, Timetables, Get } \\
\text { on and Off Public Transport }\end{array}$ & 36.63 \\
\hline $\begin{array}{l}\text { Mobility } \\
\text { Desired }\end{array}$ & $\begin{array}{l}\text { Goal is related to journey. Completion is } \\
\text { desired for journey completion. }\end{array}$ & $\begin{array}{l}\text { Confirm Information, Foursquare, Weather, } \\
\text { Track Progress }\end{array}$ & 11.63 \\
\hline $\begin{array}{l}\text { Goal } \\
\text { Essential }\end{array}$ & $\begin{array}{l}\text { Goal is unrelated to journey. Completion } \\
\text { is essential for goal completion. }\end{array}$ & $\begin{array}{l}\text { Check Emails, Make Phone Calls, Work, } \\
\text { Reminders }\end{array}$ & 20.35 \\
\hline $\begin{array}{l}\text { Goal } \\
\text { Desired }\end{array}$ & $\begin{array}{l}\text { Goal is unrelated to journey. Completion } \\
\text { is desired for goal completion. }\end{array}$ & Check Emails, Text, Facebook, Take Picture & 41.28 \\
\hline $\begin{array}{l}\text { Entertain } \\
\text { ment }\end{array}$ & $\begin{array}{l}\text { No goal per se. Activity has entertainment } \\
\text { purpose only. }\end{array}$ & $\begin{array}{l}\text { Talk, Play Games, Listen to Music, Read } \\
\text { News }\end{array}$ & 40.70 \\
\hline
\end{tabular}

In terms of social contexts, three categories were identified. Activities and behaviour of users engaging with mobile devices changed when the user was alone, with colleagues, or with friends and family. When participants were alone, the journey itself, or mobility essential tasks, occupied the individual. Individuals also carried out secondary tasks which could fall into any of the other four task categories; $27.8 \%$ were mobility desired, $27.8 \%$ were goal essential, $38.8 \%$ were goal desired, and $11.1 \%$ were entertainment related. However, during idle time participants were keen to keep busy by carrying out entertainment activities $(68 \%)$ or by pursuing goal desired activities (36.4\%).

When participants were travelling with companions, their behaviour changed according to who was travelling with them. When travelling with friends or family, the observations show that individuals engage with them most of the time. In fact, in 74\% of occurrences, participants were talking with their co-travellers. When travelling with colleagues however, social responsibilities are less prominent: Individuals talk to their colleagues $46 \%$ of the time.

Location information was also important. Ten out of 11 observed phone calls, took place in quiet or private places. Location also affected the activities that are carried out. Tasks related to work mainly occurred on trains during longer journeys and when participants were close to their destination last minutes information checks were made.

\section{THEMES OF MOBILE INTERACTIONS}

The classification of mobile interactions is based on five themes identified in this study. These themes and their impact on the classification are explored here. All names used in vignettes in Table 4 are aliases.

\subsection{Happy to Multitask?}

Multitasking of a sequential or simultaneous nature occurred in $81 \%$ of the observed interactions. So users juggle a number of activities when using personal mobile devices. This is also highlighted by the participant Kate, who states that "I spend most of my time doing two things at the same time. And that's just the way I survive." Participants also used coping strategies when multitasking, such as slowing down walking pace in 21 observations (see Jasmine's behaviour). Further observations also showed people using their devices briefly when they had a quiet moment and thereby reduced workload later. Thus users spend a large proportion of time multitasking when travelling. This reiterates the importance of using travel time wisely by increasing the positive utility, supporting journey related activities and reducing stress and anxiety.

\subsection{Less is More}

The relevance of five elements of context was examined. Participants easily adjusted to physical, temporal and technical aspects of context. First, participants, who travelled by train and did not have network access en route, focused their interactions around stations, assuming they would have access then. Secondly, although physical changes in the environment such as variation in light, noise, temperature, or traffic occurred frequently during observations, the impact on the user and mobile interactions was minimal. Finally, observations showed that the time of day was not critical for interactions, but became relevant in conjunction with tasks, as the temporal situation can spark certain activities. Some participants managed delays by informing others as previously shown [19]. The interviews and the observations also reveal that individuals planned how to make use of their time, so that it was not wasted as expected by [14]. Mark and Chris used their time to prepare for meetings, while Emma confirmed a reservation.

Yet physical, temporal and technical aspects of context are commonly used in context-aware applications [e.g. $5 ; 10 ; 18]$. One major benefit of these specific elements is that they are relatively easy to sense. Therefore, information, like noise 
Table 4. Vignettes.

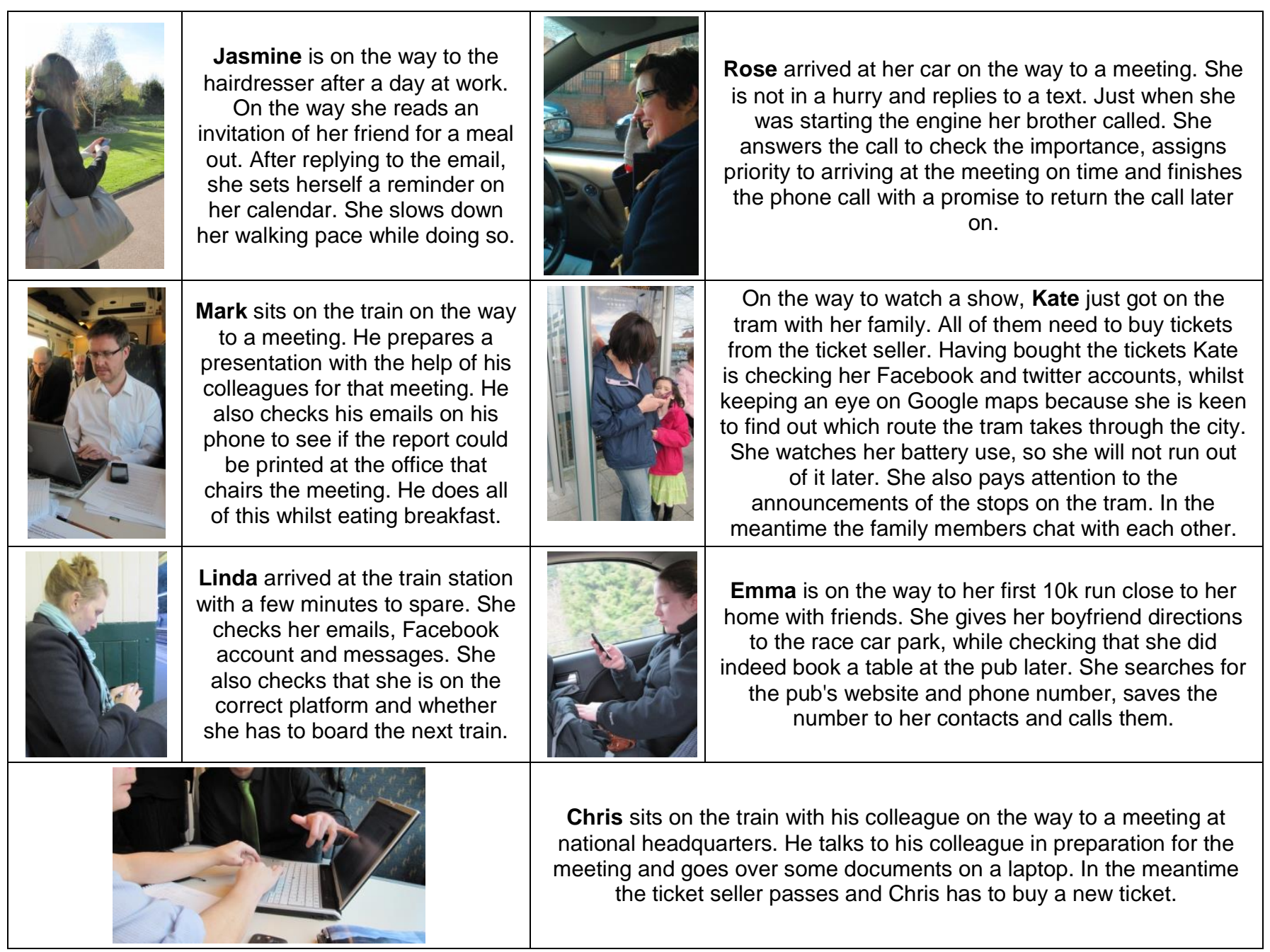

temperature, location, or orientation, is readily available. So, high quantities of data can be sensed by mobile devices. But simply because information is easily measured and there is plenty of it, does not mean it is valuable. Instead, the information should have some effect on the user interaction, it should be relevant [2]. The observation analysis implies that physical, temporal and technical facets themselves can be regarded as background information. But, having adopted an alternate view relevance is key to create impact through contextaware applications. So, rather than identifying what can be sensed, the approach here taken aims to create a classification around context that has an impact on the user during mobile interactions. This classification in turn could form the basis for understanding user behaviour in journeys. Thus, it reveals how to use environmental information to infer user contexts and provide services that have a higher impact on mobile $\mathrm{HCl}$.

\subsection{What is Actually Relevant?}

In the study, three critical elements shaping mobile contexts during end-to-end journeys were isolated.
Firstly, location information is relevant as it affects the manner of interactions. For example, calls took place in quiet or private places, as shown by Emma and Rose. Location also affects the activities that are carried out. For instance, tasks related to work mainly occurred on trains during longer journeys shown by Mark and Chris. The importance of location is recognized in most context-aware applications [e.g. 5; 18; 20]. However, it has to be noted that the main reason for this is the change of the social contextual situation within these locations. So, the relation between location and social norms and expectations makes locational information particularly valuable.

Secondly, task context influences how activities are combined and managed. For instance, when walking outside, participants restricted themselves to short precise interactions (see Jasmine). Thirdly, social context determines the type of activities that are acceptable and the amount of time that can be spent on these. Like Kate, who used her mobile phone, while her family members talked to each other. The interview shows the tensions between socials responsibilities and desired actions when 
she states that "I do use it [IPhone] when I am out but I try not to be completely antisocial. So if I am with the kids I will try and not use it too much. My husband will probably disagree and say I do use it a lot." Thus, social and task context determine the priority of activities in mobile settings and location provides a frame of reference for both. Other research also showed the role of temporal tensions, specifically the difference between states of waiting and hurrying [19]. These tensions are reflected in prioritising mobility essential tasks in states of hurrying, but carrying out goal desired or entertainment tasks when waiting (see Kate, Linda \& Rose).

\subsection{Essential Tasks Trump Desired Actions}

The study shows, people often multitask when using mobile devices during end-to-end journeys. But what strategies do they apply to manage and prioritise these activities given their setting? To illustrate the findings, a categorisation of tasks was proposed as shown in Table 3.

Using this categorisation of tasks, two observations can be made about mobile interactions in end-toend journeys. First, mobility essential tasks take priority compared to other tasks. For instance, Kate and Linda stopped all other tasks to buy a tram ticket and to check for the train. However, there is a potential that mobility essential tasks move into the background and back into focus when the user is at a decision point. Emma gave her partner, who was driving, directions to the event car park. She then switched tasks in order to check her reservation. At the next decision point, the task of providing directions moved back into focus. Moreover, entertainment tasks can be carried out at the same time when it does not interrupt the other task. For instance Chris listened to music while walking to the station. Also, Jasmine checked her emails and read a blog while listening to music. But travelling itself may not be the primary focus of the journey. Journeys usually have one purpose: get to a point $x$. But particularly on the way back this can be secondary. The mobility related tasks then have a lower priority. For instance when the participant Ed was returning from a meeting, he happily stopped walking to talk and send messages to his friends.

Secondly, goal essential tasks take priority over all tasks apart from mobility essential tasks. This is illustrated by Mark's behaviour as he prepared a presentation rather than engaging in desirable tasks. These observations show that, perhaps unsurprisingly, essential tasks trump other desired tasks when users have to manage and prioritise multiple tasks. Thus, it is critical for mobile devices to support the completion of activities related to these to reduce stress and workload while on the move. However, the prioritisation of desired tasks and entertainment is not as straight forward.

\subsection{The Importance of the Social Environment}

This research also recognises the importance of the social context in mobile interactions, as the types of activities carried out and their prioritisation are affected by it. To that extent a distinction can be made about behaviours when users are alone and when they are in the company of people.

When participants were alone, the journey itself occupied the individuals, for instance when driving or walking. Secondary tasks were also carried out which could fall into any of the other task categories. During idle time on public transport participants stayed busy by engaging in goal oriented or entertainment activities. There is no social stigma attached to carrying out tasks or to indulge in entertainment when travelling alone. Thus tasks can be managed more efficiently. To that effect shorter tasks are carried out when the individual is active or at a decision point and longer tasks are carried out when the individual is idle, while entertainment may continuously go on.

The participants' behaviour was influenced by travel companions. When travelling with friends or family, the observations show that individuals usually engage with them (see Emma). Contrary to being alone, individuals have social responsibilities which vary from being the host of the journey, like Kate, to simply being a friend. It is often considered rude to ignore the people around you. So, entertainment as a task completely changes and often comprises of talking to friends and family. However, it is also accepted that an individual can "leave" the group and pursue their own goals when appropriate. Moreover, the use of a mobile device may also be sparked by the conversation itself. Conversation with her friends caused Emma to confirm her reservation.

When travelling with colleagues however, social responsibilities are less prominent. It is much more acceptable to follow one's own goals, especially when working on journeys (see Mark \& Chris). As before, it is acceptable when an individual steps away from the group, especially for a personal interaction. But the moments in which individuals create their own space can be much more prolonged compared to those when travelling with friends and family. Also, during the return journey entertainment rises in importance.

\section{A CLASSIFICATION FOR MOBILE INTERACTIONS}

The final classification of mobile interactions is shown in Figure 1. First, the task aspect of context consists of five types of activities: mobility essential, mobility desired, goal essential, goal desired and entertainment tasks. During mobile interactions the user is mostly multitasking, 
showing the need to manage multiple tasks. To that extent, mobility and goal essential tasks are given priority over desired tasks. The study revealed that mobility essential tasks trump all other tasks, so that support of the actions and behaviour related to these becomes critical. This observation leads to two major consequences for mobile devices and interaction design. First, mobile interactions should not overload the user when mobility essential tasks are carried out. A mobile interaction that does take place simultaneously with mobility essential tasks should be important to the user and the interaction task should be delivered so that interference with mobility essential tasks is minimised. Secondly, mobile devices should be designed to support mobility essential tasks rather than interrupting them. Some basic applications, such as navigation when walking, providing information relevant to a conversation, or providing public transport information, which reveal when hurrying is required or when time is available for sidestepping, need to be revisited to suit the users' needs. This support is critical during journeys as it helps the user in uncertain situations or unfamiliar routes. When a device senses such a situation and helps with the users' decision making process stress can be reduced and the user can focus on using travel time more effectively and enjoyably.

However, the remainder of the tasks do not follow a linear hierarchy, but are influenced by the social context the user is in. When the user is alone, there are no immediate social responsibilities. At this time the potential for creating a more positive utility of travel time via context-aware systems is greatest. This can be achieved by raising the success rates of desired tasks of a professional or personal nature, or by increasing enjoyment of entertainment and relaxation. This argument is even more pertinent when the user is not engaged in mobility related tasks but is idle. However, it is important for the user to sense the signal that indicates the end of the idle waiting period, e.g. noticing the bus arrive at the stop. These are crucial events to the user's successful journey completion. Mobile interfaces may amplify these signals by adding cues.

Whilst travelling with friends and family, the social responsibilities are greatest, so that interactions with mobile devices are only acceptable when important or short. Therefore, longer, unnecessary interactions are treated with lower priority compared to entertainment. Mobile devices should only interrupt the user when interactions are necessary or brief and not disruptive to the social context. Devices could potentially use learning algorithms in order to identify interactions that should be given a higher priority than the social interaction itself, as this varies from individual to individual. In addition, designers could develop interactions that enhance the social context, for
Figure 1: Classification of Mobile Interactions

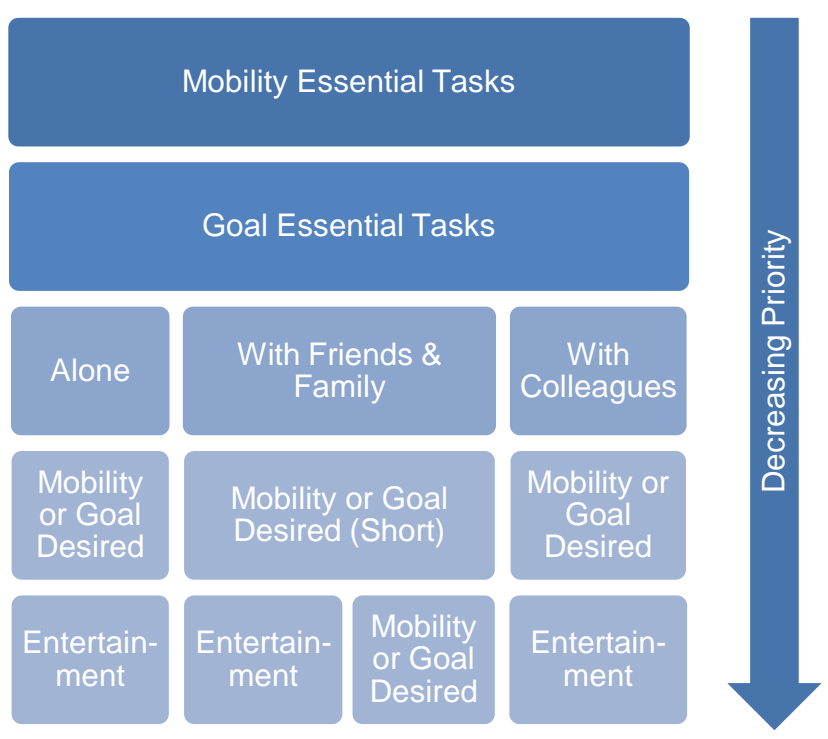

instance creating opportunities for sharing data like pictures, cinema times, or to answer questions or queries that arise in discussions, and so on. This would lead to increased utility of travel time via higher support for mobility related activities when necessary and assistance in desired activities as well as entertainment when possible.

\section{DISCUSSION}

The classification of mobile interactions developed in this paper is a key stepping stone for sensing and predicting user contexts for mobile interactions by investigating which aspects of context are critical to the user when completing tasks on the move. These insights in turn can be used by designers, $\mathrm{HCl}$ practitioners and researchers to create context-aware interfaces that allow users to increase the positive utility of travel time. To close the loop, the classification is discussed in light of the observations made in the introduction.

First, there are many opportunities for mobile device use during journeys. Primarily, travellers often multitask by combining journey related activities with activities such as communications, entertainment or recreation, household or personal activities [12]. Additionally, users are faced with a number of unaddressed challenges that contextaware systems could overcome. These challenges include the need or desire for multitasking, management of interruptions or unpredicted incidents, and additional distractions as well as the fluctuation of settings [1]. The ever increasing amount of time spent travelling highlights the need to use this time effectively by increasing positive travel time utility. Kenyon \& Lyons [12] suggest further that the ability to multitask can influence the attractiveness of travel as an accessibility option 
and as an enabler for a secondary task. This can be supported by context-aware systems that use the classification of mobile interactions in three ways. First, the classification revealed the importance of mobility essential tasks, which need to be supported to reduce stress and increase effective and timely decision making. Currently, the attention of users is often pulled to other goal desired or entertainment tasks. Secondly, mobile devices have the potential to increase the user's ability to multitask for instance by the use of multimodal devices that could use contextual information to trigger appropriate modalities. Finally, mobile devices could increase the support for desired tasks and entertainment or relaxation especially when the user is idle.

Secondly, it is clear that mobile interactions cannot be investigated without considering the notion of context. It affects people's actions and it also allows for interpretation and understanding of those actions [7]. While most research and development surrounding context treats context as a representational problem, which means it can be known, represented and perceived by technology $[7 ; 15]$. A better fit for the results from this paper is an alternative view where context is treated as an interactional problem as context does not reflect absolute truths, but it is subject to convention, human perception, and social experience [15]. Dourish [7] also argues that context does not simply exist, but it is actively created and managed. This alternative view of context is more valuable than its counterpart as it addresses the notion of relevancy.

Although an increasing number of context-aware applications are built [e.g. 5; 10; 18], only a rather small share use task and social contextual information [e.g. 8; 16]. Today, devices can collect a high range and quantity of data to support this type of contextual awareness. But less information may be more valuable providing it is relevant and therefore it can be used to accurately predict the user context. A device that collects too much information uses power and resources less effectively and may overburden the user with information that is not essential. The classification proposed here revealed that task and social contextual aspects are most relevant to the user because these factors influence their actions and interactions with mobile devices.

\section{CONCLUSION}

The classification developed is valuable to designers, $\mathrm{HCl}$ practitioners and researchers for three reasons. Firstly, the classification identified that task, social, and locational elements of context are relevant for mobile interactions in end-to-end journeys. The classification also demonstrates that mobile interactions do tend to fall into meaningful categories from which task priorities can be inferred. While mobility and goal essential tasks are prioritised, the prioritisation of the remaining three tasks - mobility and goal desired tasks and entertainment - is determined by the social context.

Secondly, this work argues that sensing less environmental data leads to more valuable applications as the focus should be to sense relevant information. This classification provides guidance to inform designs of mobile applications that allow inference of context in an efficient manner by pinpointing the need for specific contextual information.

In the future, mobile sensing data could also be used to reinforce and develop this classification to build up extensive knowledge of task priorities and types and thus enable devices to automatically determine the nature of interaction most appropriate for a given point in a journey.

The journey is increasingly becoming part of the experience of an individual, whether it is travel to an event or as part of the working day. Mobile technologies offer the potential to allow people to use the journey, whether it is to enhance their enjoyment of the event or to allow them to achieve more in their professional and personal lives. In these situations such a classification offers a sound basis for mobile application designers to understand the nature of task goals and the appropriate types of interactions that should be proposed.

\section{ACKNOWLEDGEMENTS}

This work is supported by the Horizon Doctoral Training Centre (RCUK Grant No. EP/G037574/1) and the Horizon Digital Hub (RCUK Grant No. EP/G065802/1) at the University of Nottingham.

\section{REFERENCES}

1. Barnard, L., Yi, J.S., Jacko, J.A. \& Sears, A. (2007) Capturing the Effects of Context on Human Performance in Mobile Computing Systems, Personal Ubiquitous Computing, 11(2), 81-96.

2. Bradley, N.A. \& Dunlop, M.D. (2005) Toward a Multidisciplinary Model of Context to Support Context-Aware Computing, Human-Computer Interaction, 20, 403-466.

3. Denzin, N.K. \& Lincoln, Y.S. (2000), Handbook of Qualitative Research $\left(2^{\text {nd }}\right.$ ed). Sage Publications, London.

4. Dey, A.K. (2001) Understanding and Using Context, Personal and Ubiquitous Computing, 5(1), 4-7. 
5. Dey, A.K. \& Abowd, G.D. (2001) CybreMinder: A Context-Aware System for Supporting Reminders, Lecture Notes in Computer Science, 1927, 201-207.

6. Dourish, P. (2001) Seeking a Foundation for Context-Aware Computing, Human-Computer Interaction, 16, 229-241.

7. Dourish, P. (2004) What We Talk About When We Talk About Context, Personal Ubiquitous Computing, 8(1), 19-30.

8. Esbjörnsson, M., Juhlin, O. \& Östergen, M. (2003), Motorcycling and Social Interaction: Design for the Enjoyment of Brief Traffic Encounters, GROUP'03, Sanibel Island (FL), Nov 9-12, 354-356. ACM Press, New York.

9. Froehlich, J., Chen, M.Y., Consolvo, S., Harrison, B. \& Landay, J.A. (2007), MyExperience: A System for In situ Tracing and Capturing of User Feedback on Mobile Phones, MobiSys 2007, San Juan, June 11-13. ACM Press, New York.

10. Håkansson, M., Gaye, L., Ljungblad, S., \& Holmquist, L.E. (2006), More than Meets the Eye: An Exploratory Study of Context Photography, NordiCHI 2006, Oslo, Oct 14-18, 262-271. ACM Press, New York.

11. Jumisko-Pyykkö, S. \& Vainio, T. (2010) Framing the Context of Use for Mobile $\mathrm{HCl}$, International Journal of Mobile Human Computer Interaction, 2(4), 1-28.

12. Kenyon, S. \& Lyons, G. (2007) Introducing Multitasking to the study of travel and ICT: Examining its extent and assessing its potential importance, Transportation Research Part A, 41, 161-175.
13. Lyons, G. \& Hatman, R. (2002) The UK Public Transport Industry and Provision of Multi-Modal Traveller Information, International Journal of Transport Management, 1, 1-13.

14. Mokhtarian, P.L. \& Salomon, I. (2001) How Derived is the Demand for Travel? Some Conceptual and Measurement Considerations, Transport Research Part A, 35, 695-719.

15. Oulasvirta, A., Tamminen, S. \& Höök, K. (2005) Comparing Two Approaches to Context: Realism and Constructivism, CC 2005, Edinburgh, April 4-8, 195-198. ACM Press, New York.

16. Shin, C., Hong, J.-H. \& Dey, A.K. (2012) Understanding and Prediction of Mobile Application Usage for Smart Phones, UbiComp'12, Pittsburgh, USA, Sep 5-8, 173182. ACM Press, New York.

17. Sohn, T., Li, K.A., Grisworld, W.G. \& Hollan, J.D. (2008) A Diary Study of Mobile Interaction Needs, CHI 2008, Florence, Italy, Apr 5-10, 433442, ACM Press, New York.

18. Smith, D. Ma, L. \& Ryan, N. (2006) Acoustic Environment as an Indicator of Social and Physical Context, Personal Ubiquitous Computing, 10, 241-254.

19. Tamminen, S., Oulasvirta, A., Toiskallio, K. and Kankainen, A. (2004) Understanding Mobile Contexts, Personal Ubiquitous Computing, 8(2), 135-143.

20. Yoon, Y., Ahn, Y., Lee, G., Hong, S. and Kim, M. (2008) Context-Aware Photo Selection for Promoting Photo consumption on a Mobile Phone, Mobile HCl 2008, Amsterdam, Sep 2-5, 33-42. ACM Press, New York. 